Каталитическое фотометрическое определение нитрат-ионов с использованием циклического инжекционного анализа

\author{
В.М. Захаренко \\ 1ЗАО НПО «Гранит-НЭМП» 191014, Российская Федерация, е. Санкт-Петербург, \\ ул. Госпитальная, 3 \\ ${ }^{2}$ СПбГУ, Химический фракультет, 198504, Российская Федерация, г. Санкт-Петербург, \\ Университетский пр., 26
}

*Адрес для переписки: Худяков Юрий Сергеевич , E-mail: uhs83@mail.ru

Поступила в редакцию 18 декабря 2014 г., после исправлений - 26 января 2015 г.

\begin{abstract}
Разработана кинетическая фотометрическая методика экспрессного определения нитратионов в природных пресных и соленых водах с использованием циклического инжекционного анализа. Методика основана на каталитическом влиянии нитрит-ионов на реакцию окисления этилендиаминтетраацетата марганца (II) пероксидом водорода после предварительного восстановления нитрат-ионов до нитрит-ионов при помощи омедненного Cd-редуктора. В результате реакции образуется окрашенное соединение - этилендиаминтетраацетат марганца (III). Измерение оптической плотности раствора проводили при длине волны 520 нм. Диапазон определяемых концентраций 0.1-5 мг/л. Предел обнаружения 0.02 мг/л. Относительная случайная погрешность определения не превышает $15 \%$.

Рассмотрены преимущества и недостатки расположения омедненного Cd-редуктора в гидравлических схемах проточно-инжекционного и циклического инжекционного анализов. Рассмотрен представленный в литературе вариант расположения омедненного Cd-редуктора в методике, реализованной в циклическом инжекционном анализе, и предложен альтернативный вариант размещения. Проведена проверка методики определения нитрат-ионов в пресной и соленой воде методом добавок и референтным методом. Основными преимуществами данной методики являются избирательность, экономия используемых реактивов и автоматически настраиваемая частота проведения анализов. Методика может быть использована для автоматического определения нитрат-ионов в природной воде в непрерывном режиме с максимальной производительностью 12 анализов/ч, а также в режиме с меньшей производительностью, с установленным перерывом между анализами.

Ключевые слова: нитрат-ионы, фотометрическое определение, каталитическая реакция, вода, циклический инжекционный анализ.
\end{abstract}

For citation: Analitika i kontrol' [Analytics and Control], 2015, vol. 19, no. 1, pp. 77-84. DOI: 10.15826/analitika.2015.19.1.012

\title{
Catalytic photometric determination of nitrate in nature water using stepwise injection analysis
}

\author{
V.M. Zakharenko', Iu.S. Khudiakov1", A.V. Mozzhukhin', A.L. Moskvin ${ }^{2}$ \\ 'ZAO NPO Granit-NEMP, ul. Gospital'naia, 3, Saint Petersburg, 191014, Russian Federation \\ ${ }^{2}$ Department of Analytical Chemistry, Faculty of Chemistry, Saint Petersburg State University, pr. \\ Universitetskii, 26, Saint Petersburg 198504, Russian Federation
}

*Corresponding author: Iuriy S. Khudiakov, E-mail: uhs83@mail.ru

Submitted 18 December 2014, received in revised form 26 January 2015

A new express technique for the kinetic catalytic photometric determination of nitrate by stepwise injection analysis is introduced. The detection is based on the catalytic effect of nitrite on the oxidation of ethylendiaminetetreaacetat manganese (II) to red ethylendiaminetetreaacetat manganese (III) by hydrogen peroxide. Nitrate was preliminary reduced to nitrite using copperised cadmium reductor column. The measurement of the red color absorbance was monitored at $520 \mathrm{~nm}$ wavelength. Determined the reliable working concentration range to be $0.1-5 \mathrm{mg} / \mathrm{dm}^{3}$ with $3 \sigma$ detection limit of $0.02 \mathrm{mg} / \mathrm{dm}^{3}$. The relative random measurement error of the technique does not exceed $15 \%$. The advantages and disadvantages of the 
copperised cadmium reductor location in the hydraulic circuits of the flow-injection and stepwise injection analysis methods were considered. Reviewed the copperised cadmium reductor placing using the stepwise injection analysis method presented in the literature and offered another alternative of placing. The proposed technique was tested by the added - found method and referent method with satisfactory results. The major advantages of this technique are simplicity, selectivity and economy of reagents. The technique can be used to automatically determine nitrate in continuous mode at a rate of 12 samples per hour, and in the mode with a delay between samples. The technique could be applied successfully for the nitrate determination in nature fresh and salt waters.

Key words: nitrate, photometric determination, catalytic reaction, nature water, stepwise injection analysis.

\section{Введение}

Диапазон содержания нитрат-ионов в природной воде не превышает нескольких миллиграмм на литр и их наименьшая концентрация наблюдается в зимний, а наибольшая - в летний период, когда повышена солнечная активность и активность фитопланктона [1]. Несмотря на то, что нитрат-ионы являются умеренно-опасным веществом (3 класс опасности) и допускаются их высокие содержания - предельно-допустимая концентрация для вод хозяйственно-питьевого и культурно-бытового назначения (ПДКв) составляет 45 мг/л [2], в связи с масштабным применением азотных удобрений в сельскохозяйственной деятельности возможно серьезное загрязнение рек и водоемов, расположенных вблизи аграрного комплекса. Регулярное употребление в пищу воды, содержащей повышенные содержания нитрат-ионов, вызывает метгемоглобинемию и, как следствие, отклонение развития головного мозга, что особенно опасно для детей грудного возраста [3, 4]. В практике экологического контроля важно определять концентрацию нитрат-ионов не только в области ПДКв, но и на уровне фоновых значений. Как правило, для водоемов известны сезонные колебания содержания нитратионов и их увеличение может свидетельствовать о наличии антропогенного источника загрязнения.

Разработано большое количество методик, направленных на измерение концентрации нитратионов в воде. Общепринятыми методиками определения являются ИСО 7890-1:2003, ИСО 7890-2:2003 и ИСО 7890-3:2006, основанные на прямом взаимодействии нитрат-ионов с 2,6-диметилфенолом, 4-фрторфенолом и сульфосалициловой кислотой соответственно с образованием окрашенных продуктов реакции [5]. Методики предназначены для лабораторного определения нитрат-ионов. Наиболее распространены методики косвенного определения нитрат-ионов после их предварительного восстановления до нитрит-ионов. В экологическом анализе такой подход оправдан, поскольку концентрация нитрат-ионов в природной воде как правило превышает концентрацию нитрит-ионов в десятки, и даже в сотни раз [6-8], поэтому концентрацией нитрит-ионов, присутствующих в природной воде, чаще всего можно пренебречь. После этапа предварительного восстановления для определения нитрат-ионов широко используют реактив Грисса-Илосвая [9-13]. Недостатками данного реакти- ва являются токсичность и низкая устойчивость в растворе, который рекомендуется использовать в день приготовления. Эти недостатки делают работу с реактивом Грисса-Илосвая неудобной в подвижных лабораториях, используемых для экспрессного анализа пробы воды на месте отбора. Альтернативой реакции с реактивом Грисса-Илосвая являются окислительно-восстановительные реакции, катализируемые нитрит-ионами. Известно, что каталитические методики обладают гораздо большей чувствительностью и часто также избирательны. Так, в ряде работ определение нитрит- и нитрат-ионов после восстановления основано на каталитическом влиянии нитрит-ионов на реакции окисления броматом калия кристаллического фииолетового [7], пирогаллолового красного [14], пирогаллолосульфонефталеина [15] и галлоцианина [16]. Диапазоны определения концентраций нитрат-ионов и пределы обнаружения (ПО) указанных реакций составили 0.003-0.15 мг/л (0.001 мг/л) [7], 0.03-2 мг/л (0.01 мг/л) [14], 0.004-0.1 мг/л (0.003 мг/л) [15] и 0.02$3.5 \mathrm{мг/л} \mathrm{(0.002} \mathrm{мг/л)} \mathrm{[16].}$

Современное развитие экологического мониторинга водных объектов основано на применении экспрессных методов анализа. Одним из самых распространенных является проточно-инжекционный анализ (ПИА) [17], который позволяет проводить определение исследуемых компонентов непосредственно на борту экологического судна в режиме on-line. По причине непрерывного потока большинства растворов, в методе ПИА наблюдаются относительно большой расход реактивов и, соответственно, образование большого количества слива. В методе циклического инжекционного анализа (ЦИА) реализован постадийный отбор аликвот пробы и реагентов [18], что позволяет по отношению к методу ПИА в 5-6 раз сократить объемы используемых растворов и объем слива, что немаловажно в условиях компактной судовой лаборатории. Кроме того, в случае отсутствия необходимости проводить анализ на максимальной скорости, в методе ЦИА возможно в автоматическом режиме введение паузы между анализами, что приводит к еще большей экономии реактивов и уменьшению объема слива.

В настоящей работе нами предложена методика циклического инжекционного определения нитрат-ионов в природных пресных и соленых водах, основанная на чувствительной каталитической реакции окисления этилендиаминтетраацетата (ком- 
плексонат) марганца (II) до комплексоната марганца (III) пероксиазотистой кислотой - продуктом взаимодействия $\mathrm{H}_{2} \mathrm{O}_{2}$ с нитрит-ионами [19], полученными на стадии предварительного восстановления нитрат-ионов с использованием омедненного Cdредуктора (Cd-редуктор).

\section{Экспериментальная часть}

Для реализации методики использовали анализатор проточно-инжекционный ПИАКОН-03 производства ЗАО НПО «Гранит-НЭМП», оборудованный восьмиходовым соленоидным краном (Cole-Parmer, США) (рис. 1).

Использовали следующие реагенты: $\mathrm{HCl}$ («Х.ч.»), $\mathrm{H}_{2} \mathrm{O}_{2}$ (медицинская), $\mathrm{MnCl}_{2} \cdot 4 \mathrm{H}_{2} \mathrm{O}$ («ч.д.а.»), этилендиаминтетраацетата динатриевая соль (комплексон III) («ч.д.а.»), $\mathrm{H}_{2} \mathrm{SO}_{4}$ («ос.ч.»), $\mathrm{CuSO}_{4} \cdot 5 \mathrm{H}_{2} \mathrm{O}$ («ч.д.а.»), $\mathrm{NaCl}$ («х.ч.»), $\mathrm{MgSO}_{4}$ («х.ч.») кадмий гранулированный (Merck, Германия), ГСО нитрат-ионов 1.0 г/л (ГСО 6696-93). Все растворы готовили с использованием дистиллированной воды.

В качестве растворов реагентов использовали $0.3 \%$ раствор $\mathrm{H}_{2} \mathrm{O}_{2}$ в $0.001 \mathrm{M} \mathrm{H}_{2} \mathrm{SO}_{4}$ и раствор комплексоната марганца (II), который готовили растворением при нагревании 10 г комплексона III в 150 мл воды, с последующим растворением в нем 200 г $\mathrm{MnCl}_{2} \cdot 4 \mathrm{H}_{2} \mathrm{O}$ при кипячении. Охлажденный раствор фильтровали в мерную колбу вместимостью 500 см³ $^{3}$ доводили до метки и перемешивали.

Корректирующий раствор готовили растворением 0.74 г комплексона III в 1 л раствора $\mathrm{HCl}$ с концентрацией $1.25 \cdot 10^{-3}$ М [1].

Основной раствор имитатора солености $100 \%$ готовили растворение 83 г $\mathrm{NaCl}$ и 17 г $\mathrm{MgSO}_{4}$ в 1 л дистиллированной воды. После приготовления раствор фильтровали. Рабочие растворы имитатора солености 5, 15, 25 и $35 \%$ готовили разбавлением исходного раствора дистиллированной водой.

Cd-редуктор представлял собой колонку длиной 30 мм и диаметром 4 мм, заполненную гранулированным кадмием. Перед использованием редуктор активировали последовательным промыванием $5 \mathrm{M} \mathrm{HCl}$ и раствором, содержащим $\mathrm{CuSO}_{4} \cdot 5 \mathrm{H}_{2} \mathrm{O}$ и комплексон III в концентрациях по 1 г/л. Последний раствор в редукторе оставляли на 12 ч, после чего редуктор был готов к использованию.

\section{Результаты и обсуждения}

\section{Размещение $\mathrm{Cd}$-редуктора в гидравлических схемах методов ПИА и ЦИА}

В качестве быстрого и эффрективного восстановителя нитрат-ионов до нитрит-ионов в потоке наиболее часто используют Cd-редуктор. Известно, что $\mathrm{Cd}$-редуктор имеет ограниченный срок устойчивой работы от нескольких часов до нескольких дней, после чего его эфрфективность снижается от анализа к анализу $[1,20]$. Введение в процесс ана-

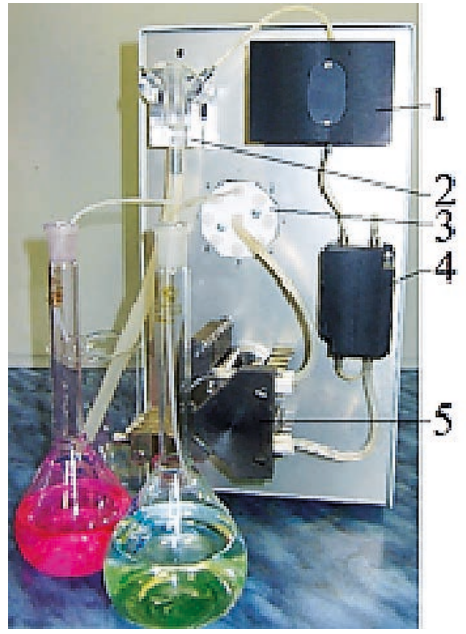

Рис. 1. Анализатор проточно-инжекционный ПИАКОН-03, оборудованный восьмиходовым соленоидным краном: 1 - фотометрический детектор; 2 - блок удаления пробы; 3 - восьмиходовой кран; 4 - термостат; 5 - перистальтический насос

лиза промывки редуктора раствором, содержащим комплексон III в кислой среде, существенно продлевает срок службы Cd-редуктора, до нескольких недель [1]. В методе ПИА за счет непрерывного протекания растворов по индивидуальным каналам возможно так скоммутировать потоки, чтобы Cd-редуктор промывался корректирующим раствором в процессе анализа.

В методе ЦИА используется одна жидкостная линия, по которой осуществляется поочередная подача растворов. Поэтому одновременная подача двух растворов и их смешение в потоке нереализуемо, в результате чего требуется введение дополнительной камеры, в которую все растворы нужно по очереди ввести и перемешать, что значительно увеличивает время анализа. В работе [13] предложена гидравлическая схема, в которой в реакционную емкость поочередно отбираются необходимые растворы, в том числе проба и корректирующий раствор. Затем смесь растворов направляется в Cd-редуктор, за которым располагается вспомогательная емкость. Из вспомогательной емкости смесь растворов с восстановленными нитрат-ионами до нитрит-ионов перемещается обратно в реакционную емкость, откуда подается в детектор.

Постадийное выполнение всех операций дает возможность в методе ЦИА индивидуально подойти к настройке каждой операции, но заметно увеличивает продолжительность анализа. Особенно сильное увеличение времени анализа, в 2-3 раза по сравнению с аналогичными методиками, реализованными методом ПИА, происходит при введении специальных процедур пробоподготовки, к которым, в данном случае, относится восстановление нитрат-ионов с помощью Cd-редуктора. Поэтому поиск оптимальных решений по проведению анализа методом ЦИА является на сегодняшний день актуальной задачей. 


\section{Аэро-гидравлическая схема}

В настоящей работе нам удалось сократить время определения нитрат-ионов на $40 \%$ с 500 с [13] до 300 с за счет совмещения реакционной емкости с кюветой детектора, переноса корректирующего раствора на линию, на которой располагается Cd-редуктор и за счет применения чувствительной каталитической реакции. Аэро-гидравлическая схема циклического инжекционного определения концентрации нитрат-ионов в природных водах представлена на рис. 2.

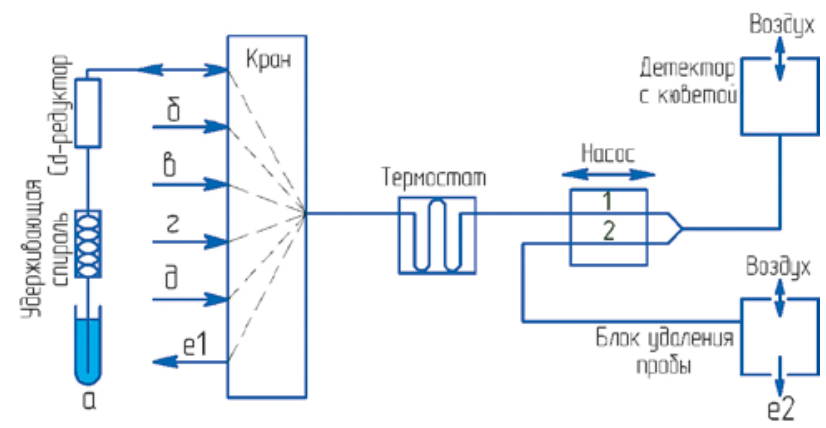

Рис. 2. Аэро-гидравлическая схема для определения нитрат-ионов в природных водах: а - корректирующий раствор; б - проба; в - комплексонат марганца (II); г $-\mathrm{H}_{2} \mathrm{O}_{2}$; д-воздух; е1, е2 - слив

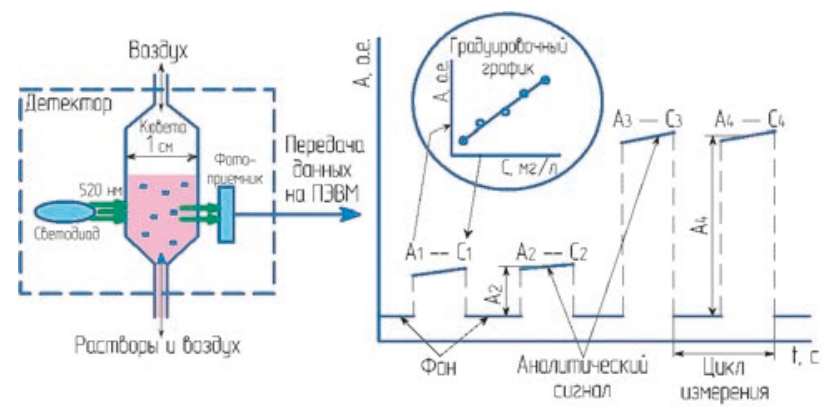

Рис. 3. Процесс измерения и обработки аналитического сигнала

Определение концентрации нитрат-ионов происходит следующем образом. Проба (б) по каналу 1 проходит через кран, термостат, насос и подается в кювету фотометрического детектора, где проводится измерение при длине волны 520 нм оптической плотности раствора, соответствующей фоновому значению концентрации нитрат-ионов (рис. 3). После реверса насоса и переключения крана в положение (е1), содержимое кюветы направляется на сброс (e1, е2). Затем в термостат отбирается проба (б), кран переключается в положение (а) и проба для восстановления нитрат-ионов направляется в Cd-редуктор и далее в удерживающую спираль, выполняющую роль буферной емкости для предотвращения попадания пробы в емкость с корректирующим раствором (а). После пропускания всей отобранной в канал 1 пробы через Cd-редуктор насос переключается и проба подается в кювету детектора, еще раз проходя через Cd-редуктор, при этом корректирующий раствор заполняет удерживающую спираль и Cd-редуктор. Далее в кювету подаются раствор комплексоната марганца (II) (в), раствор $\mathrm{H}_{2} \mathrm{O}_{2}($ (г), при постоянной подаче воздуха по каналу 2 для тщательного перемешивания. После всех растворов подается воздух (д), осуществляющий полное вытеснение всех растворов из канала 1 в кювету. В это же время протекает каталитическая реакция. Далее проводится измерение оптической плотности раствора, соответствующей концентрации нитрат-ионов в пробе. После реверса насоса и переключения крана в положение (е1), содержимое кюветы направляется на сброс (e1, е2). Затем кювета последовательно промывается корректирующим раствором (a), что позволяет в то же время восстановить активность Cd-редуктора, и пробой (б), после чего цикл измерений повторяется.

Измеренные оптические плотности фонового раствора и раствора, в котором прошла химическая реакция, оцифровываются и передаются на персональную электронно-вычислительную машину (ПЭВМ). На мониторе ПЭВМ в режиме реального времени в координатах оптической плотности $(A$, о.е.) от времени $(t, c)$ отображаются уровни измеренных значений фонового и аналитического сигналов. Программа управления анализатором ПИАКОН-03 вычисляет разницу между усредненными значениями аналитического и фонового сигналов и по заранее построенной градуировочной зависимости вычисляет концентрацию нитрат-ионов. На рис. 3 схематично показан процесс измерения оптической плотности раствора и цифровой обработки аналитического сигнала на ПЭВМ.

При наблюдении в течение 10 дней за эффрективностью работы Cd-редуктора, расположенного в соответствии с рис. 2, изменений интенсивности аналитических сигналов при прочих равных условиях не обнаружено. Данный факт позволяет сделать вывод о стабильной работе предложенной схемы.

\section{Выбор оптимальных условий}

Вследствие того, что концентрации марганца (II) и $\mathrm{H}_{2} \mathrm{O}_{2}$ сильно влияют на скорость протекания каталитической реакции, нами были исследованы зависимости оптической плотности раствора от концентрации марганца (II) и $\mathrm{H}_{2} \mathrm{O}_{2}$ при прочих постоянных условиях. Было выявлено, что повышение концентрации марганца (II) приводит к увеличению как аналитического сигнала, так и фонового. Нами выбрана такая концентрация марганца (II), при которой разница между аналитическим и фоновым сигналами была максимальной - 2 М. Максимальные значения оптической плотности раствора наблюдались в диапазоне концентраций $\mathrm{H}_{2} \mathrm{O}_{2}$ 0.30-0.35\%, при концентрации $\mathrm{H}_{2} \mathrm{O}_{2}$ менее $0.30 \%$ и более $0.35 \%$ наблюдалось уменьшение оптической плотности. Нами выбрана оптимальная концентрация $\mathrm{H}_{2} \mathrm{O}_{2}-0.30$ \%. Объемы дозирования пробы, комплексоната марганца (II) и $\mathrm{H}_{2} \mathrm{O}_{2}$ подбирали экс- 
Таблица 1

Матрица последовательности действий анализатора ПИАКОН-03 для определения концентрации $\mathrm{NO}_{3}^{-}$ в пробе.

\begin{tabular}{|c|c|c|c|c|}
\hline Этап & Время, с & $\begin{array}{c}\text { Положение } \\
\text { крана }\end{array}$ & $\begin{array}{c}\text { Направление вра- } \\
\text { щения насоса }\end{array}$ & $\begin{array}{c}\text { Скорость пода- } \\
\text { чи растворов, } \\
\text { мл/мин }\end{array}$ \\
\hline \multirow{3}{*}{ Промывка кюветы пробой } & 25 & 6 & $\rightarrow$ & 5 \\
\hline & 15 & д & $\rightarrow$ & 5 \\
\hline & 14 & $\mathrm{e} 1$ & $\leftarrow$ & 5 \\
\hline \multirow{2}{*}{ Подготовка фонового раствора } & 30 & 6 & $\rightarrow$ & 5 \\
\hline & 2 & e1 & - & 0 \\
\hline Измерение сигнала & 7 & $\mathrm{e} 1$ & - & 0 \\
\hline Слив & 14 & $\mathrm{e} 1$ & $\leftarrow$ & 5 \\
\hline \multirow{2}{*}{$\begin{array}{l}\text { Отбор пробы и пропускание через Cd- } \\
\text { редуктор }\end{array}$} & 9 & 6 & $\rightarrow$ & 5 \\
\hline & 40 & a & $\leftarrow$ & 1,5 \\
\hline \multirow{5}{*}{ Подготовка аналитической фрормы } & 37 & $\mathrm{a}$ & $\rightarrow$ & 2 \\
\hline & 5 & $\mathrm{~B}$ & $\rightarrow$ & 4,3 \\
\hline & 5 & $r$ & $\rightarrow$ & 1,4 \\
\hline & 20 & д & $\rightarrow$ & 5 \\
\hline & 2 & $\mathrm{e} 1$ & - & 0 \\
\hline Измерение сигнала & 7 & $\mathrm{e} 1$ & - & 0 \\
\hline Слив & 14 & $\mathrm{e} 1$ & $\leftarrow$ & 5 \\
\hline \multirow{3}{*}{$\begin{array}{l}\text { Промывка кюветы и Cd-редуктора кор- } \\
\text { ректирующим раствором }\end{array}$} & 25 & $\mathrm{a}$ & $\rightarrow$ & 5 \\
\hline & 15 & д & $\rightarrow$ & 5 \\
\hline & 14 & $\mathrm{e} 1$ & $\leftarrow$ & 5 \\
\hline
\end{tabular}

Примечания: « $\rightarrow », ~ « \leftarrow »-$ направление движения растворов в сторону детектора и от детектора; «-» - остановка насоса.

периментально и они составили 0.75 мл, 0.36 мл и 0.12 мл соответственно. Также в кювету вводилось 0.33 мл корректирующего раствора для количественного вытеснения пробы из удерживающей спирали и Cd-редуктора. Скорость пропускания пробы через Cd-редуктор составляла 1.5 мл/мин при первом пропускании и 2 мл/мин при втором. Длина удерживающей спирали 180 см, внутренний диаметр 1 мм. Кювета выполняла функцию реакционной емкости и имела цилиндрическую форму с рабочим объемом 3.0 мл и минимальным объемом для измерений 1.5 мл, длина оптического пути 1 см. В проточном термостате поддерживалась постоянная температуpa $30{ }^{\circ} \mathrm{C}$. Для реализации методики была составлена матрица последовательности манипуляций для анализа, которая приведена в табл. 1.

По каналам 1 и 2 (рис. 2) установлены силиконовые трубки для перистальтического насоса с внутренним диаметром 1 и 2 мм соответственно. По каналу 2 скорость слива растворов и подачи воздуха не нормировались и были максимальными.

В методе ЦИА измерение аналитического сигнала проводится в неравновесных условиях, а в условиях протекания химической реакции, что отображено на рис. 3 в виде увеличения оптической плотности во время измерения. Но, благодаря программному управлению анализатором ПИАКОН-03, соблюдается строгая воспроизводимость заложенных в матрицу операций, поэтому можно говорить об измерении сигнала в квазистационарных условиях.
По окончании этапа цикла возможны два варианта в зависимости от задачи, решаемой с помощью анализатора:

- анализатор в цикле возвращается к первому этапу и цикл повторяется неограниченное число раз. Данный вариант подходит для непрерывного анализа со скоростью 12 проб/час;

- включается таймер, заложенный в программе, и возвращение к этапу промывки пробой происходит с заданной задержкой. Данный вариант подходит для постов контроля качества воды в конкретной точке забора пробы.

\section{Градуировочный график}

Градуировочная зависимость оптической плотности А от концентрации нитрат-ионов $C$, мг/л $(A=0.253 C+0.011)$ линейна в диапазоне измеряемых концентраций 0.1-5 мг/л. Предел обнаружения по критерию «трех сигма» $\left(n_{\text {фон }}=15, P=0.997\right)$ 0.02 мг/л. Погрешность определения не превышает $15 \%$. Проверка градуировочного графика по контрольным растворам показала следующие результаты. При введении 0.50, 0.60, 2.00 и 4.00 мг/л, было получено $0.49 \pm 0.01,0.59 \pm 0.04,2.12 \pm 0.04$ и $4.09 \pm 0.08 \mathrm{мг/л.} S(n=5, P=0.95)$ составило 0.01 , $0.03,0.03$ и 0.06 соответственно. При построении градуировочного графика и его контроля растворы готовили на дистиллированной воде с использованием ГСО нитрат-ионов. 
Таблица 2

Влияние солености на протекание химической реакции

\begin{tabular}{|c|c|c|c|c|c|}
\hline Соленость, \%о & 0 & 5 & 15 & 25 & 35 \\
\hline $\begin{array}{l}\text { Измеренная концентрация нитрат-ио- } \\
\text { нов, мг/л }\end{array}$ & $2.02 \pm 0.10$ & $2.05 \pm 0.10$ & $2.10 \pm 0.11$ & $2.15 \pm 0.09$ & $2.18 \pm 0.14$ \\
\hline$S(n=5, P=0.95)$ & 0.08 & 0.08 & 0.09 & 0.07 & 0.11 \\
\hline $\begin{array}{l}\text { Отклонение от реальной концентрации } \\
\text { нитрат-ионов, \% }\end{array}$ & 1.0 & 2.5 & 5.0 & 7.5 & 9.0 \\
\hline
\end{tabular}

\section{Влияние мешающих компонентов}

Основными мешающими компонентами являются нитрит-ионы в молярном отношении к нитрат-ионам $0.6: 1$ и соленость. Проверку влияния солености на протекание химической реакции проводили на модельных растворах с различной соленостью с применением градуировочной зависимости, построенной по стандартным растворам нитрат-ионов в дистиллированной воде. В табл. 2 представлены результаты измеренных значений концентрации нитрат-ионов при исходной концентрации 2 мг/л в зависимости от солености воды.

Максимальное отклонение от реальной концентрации нитрат-ионов в 9 \% находится в пре- делах установленной для предлагаемой методики погрешности определения $15 \%$, что позволяет говорить о незначимом влиянии солености на величину определяемой концентрации. Однако, чтобы избежать получения систематической погрешности, рекомендуется проводить градуировку по стандартным растворам, приготовленным на имитаторе солености, с соленостью, соответствующей воде исследуемого региона.

Также на показания измерений влияют оксиды азота и сильные окислители, которые в природных водах не содержатся в концентрациях, мешающих реакции определения нитрат-ионов.

Таблица 3

Результаты определения нитрат-ионов в реальных объектах методом добавок.

\begin{tabular}{lccc}
\hline \multicolumn{1}{c}{ Объект анализа } & Добавка, мг/л & Найдено, мг/л & $\mathrm{S}(\mathrm{n}=5, \mathrm{P}=0.95)$ \\
\hline \multirow{2}{*}{ Артезианская вода №1 } & - & $0.13 \pm 0.01$ & 0.01 \\
& 1.00 & $1.16 \pm 0.14$ & 0.11 \\
\multirow{2}{*}{ Артезианская вода №2 } & - & $0.93 \pm 0.04$ & 0.03 \\
& 4.00 & $5.01 \pm 0.27$ & 0.22 \\
\multirow{2}{*}{ р. Нева } & - & $0.89 \pm 0.02$ & 0.02 \\
& 1.00 & $1.92 \pm 0.11$ & 0.09 \\
\hline \multirow{2}{*}{ Красное море } & 3.00 & $3.93 \pm 0.20$ & 0.16 \\
& - & $0.21 \pm 0.02$ & 0.02 \\
\end{tabular}

Примечание: «-» - добавку не вводили.

Таблица 4

Сопоставление результатов анализа, полученных предлагаемой методикой и референтной методикой.

\begin{tabular}{|c|c|c|c|c|c|}
\hline $\begin{array}{l}\text { Объект } \\
\text { анализа }\end{array}$ & $\begin{array}{c}\text { Добавка, } \\
\text { мг/л }\end{array}$ & $\begin{array}{c}\text { Предлагаемая } \\
\text { методика }\end{array}$ & $\begin{array}{l}S(n=5, \\
P=0.95)\end{array}$ & $\begin{array}{c}\text { Референтная } \\
\text { методика }\end{array}$ & $\begin{array}{l}S(n=5 \\
P=0.95)\end{array}$ \\
\hline Дистиллят & 4.00 & $3.95 \pm 0.14$ & 0.11 & $4.07 \pm 0.21$ & 0.17 \\
\hline Артезианская вода & - & $0.13 \pm 0.01$ & 0.01 & $0.11 \pm 0.01$ & 0.01 \\
\hline № 1 & 2.00 & $2.10 \pm 0.11$ & 0.09 & $2.05 \pm 0.23$ & 0.19 \\
\hline \multirow{2}{*}{ р. Нева } & - & $0.89 \pm 0.02$ & 0.02 & $0.92 \pm 0.04$ & 0.03 \\
\hline & 0.60 & $1.45 \pm 0.10$ & 0.08 & $1.53 \pm 0.12$ & 0.10 \\
\hline \multirow{2}{*}{ Баренцево море } & - & $0.22 \pm 0.02$ & 0.02 & $0.25 \pm 0.04$ & 0.03 \\
\hline & 3.00 & $3.18 \pm 0.20$ & 0.16 & $3.22 \pm 0.26$ & 0.21 \\
\hline
\end{tabular}

Примечание: «-» - добавку не вводили. 


\section{Проверка методики на реальных объектах.}

Возможность применения методики для исследования реальных объектов проводили на пробах артезианских вод, невской воде и на пробе воды, отобранной в Красном море, методом добавок. Данные представлены в табл. 3.

Предлагаемую методику проверяли референтным методом анализа. Для этого использовали методику выполнения измерения (МВИ) массовой концентрации нитрат-ионов в природных и очищенных сточных водах фотометрическим методом в проточно-инжекционном анализе с применением реактива Грисса-Илосвая (свидетельство об аттестации МВИ № 242/24-2007, выданное ФГУП «ВНИИМ им. Д.И.Менделеева»). Результаты анализа представлены в табл. 4.

\section{Выводы}

Разработана методика циклического инжекционного определения нитрат-ионов в природных пресных и соленых водах. Диапазон определяемых концентраций нитрат-ионов 0.1-5 мг/л. Предел обнаружения 0.02 мг/л. Время одного измерения 5 минут. Погрешность определения не превышает $15 \%$.

Предложенная методика позволяет отказаться от использования токсичного реактива ГриссаИлосвая, значительно уменьшить расход растворов и объем слива отработанных растворов. Данные преимущества имеют большое значение в практике экологического анализа.

Предложено усовершенствование схемы метода ЦИА, что позволило исключить ряд шагов и уменьшить время проведения анализа на $40 \%$.

\section{ЛИТЕРАТУРА}

1. Taniai T., Sakuragawa A., Okutani T. Monitoring of hydrogen peroxide, nitrate and nitrite in rain water // Anal. Sci. 2000. V. 16. P. $275-281$.

2. Кротов Ю.А., Карелин А.О., Лойт А.О. Предельно допустимые концентрации химических веществ в окружающей среде: под ред. Ю.А. Кротова. СПб.: Мир и семья, 2000. $360 \mathrm{c}$.

3. Camargo J.A., Alonso A. Ecological and toxicological effects of inorganic nitrogen pollution in aquatic ecosystems: A global assessment // Environ. Int. 2006. V. 32. P. 831-849. 4. Swann P.F. The toxicology of nitrate, nitrite and n-nitroso compounds // J. Sci. Food Agric. 1975. V. 26, № 11. P. 1761-1770. 5. Фомин Г.С. Вода. Контроль химической, бактериальной и радиационной безопасности по международным стандартам. Энциклопедический справочник. М.: Протектор, 2000. 849 c.

6 . Development of a sequential injection system for the determination of nitrite and nitrate in waters with different salinity: Application to estuaries in NW Portugal / R. Mesquita [et al.] // Anal. Methods. 2009. V. 1. P. 195-202.

7. Yue X-F., Zhang Z-Q., Yan H-T. Flow injection catalytic spectrophotometric simultaneous determination of nitrite and nitrate // Talanta. 2004. V. 62. P. 97-101.

8. A simple simultaneous flow injection method based on phosphomolybdenum chemistry for nitrate and nitrite deter- minations in water and fish samples / L. Monser [et al.] // Talanta. 2002. V. 57. P. 511-518.

9. Multiparametric automated system for sulfate, nitrite and nitrate monitoring in drinking water and wastewater based on sequential injection analysis / A. Ayala [et al.] // Microchem. J. 2012. V. 100. P. 55-60.

10. Field measurement of nitrate and estuarine waters with a flow analysis system on-line zinc reduction / P.S. Ellis [et al.] // Talanta. 2011. V. 84. P. 98-103.

11. Multi-pumping flow system for the determination of nitrite and nitrate in water samples / C. Pons [et al.] // Microchim Acta. 2008. V. 161. P. 73-79.

12. Automated simultaneous monitoring of nitrate and nitrite in surface water by sequential injection analysis / Z. Legnerova [et al.] // Water Research. 2002. V. 36. P. 2777-2783. 13. Булатов А.В., Ивасенко П.А., Москвин Л.Н. Циклическое инжекционное фотометрическое определение нитрит- и нитрат-ионов в водных средах при их совместном присутствии // Журн. аналит. химии. 2010. Т. 65, № 8. C. 833-837.

14. Kazemzadeh A., Ensafi A.A. Simultaneous determination of nitrite and nitrate in various samples using flow-injection spectrophotometric detection // Microchem. J. 2001. V. 69. P. 159-166.

15. Ensafi A.A., Rezael B., Nouroozi S. Simultaneous spectrophotometric determination of nitrite and nitrate by flow injection analysis // Anal. Sci. 2004. V. 20. P. 1749-1753.

16. Ensafi A.A., Kazemzadeh A. Simultaneous determination of nitrite and nitrate in various samples using flow injection with spectrophotometric detection // Anal. Chim. Acta. 1999. V. 382. P. $15-21$.

17. Шпигун Л.К. Проточно-инжекционный анализ / Журн. аналит. химии. 1990. Т. 45, № 6. С. 1045-1091.

18. Мозжухин А.В., Москвин А.Л., Москвин Л.Н. Циклический инжекционный анализ - новый метод проточного анализа // Журн. аналит. химии. 2007. Т. 62, № 5. С. 527-531. 19. Клочковский С.П., Чистота В.Д. Каталитический метод обнаружения и количественного определения нитрит-ионов и окислов азота // Сб. науч. Тр. Магнитогорск. Горно-мет. Ин-та, 1971. № 87. С. 70-72.

20. Configuration with internally coupled valves to overcome shortcomings in the simultaneous determination of nitrite and nitrate by flow-injection analysis / B. Bermudez [et al.] // Talanta. 1988. V. 35, № 10. P. 810-812.

\section{REFERENCES}

1. Taniai T., Sakuragawa A., Okutani T. Monitoring of hydrogen peroxide, nitrate and nitrite in rain water. Anal. Sci., 2000, vol. 16 , pp. 275-281.

2. Krotov lu.A., Karelin A.O., Loit A.O. Predel'no dopustimye kontsentratsii khimicheskikh veshchestv $v$ okruzhaiushchei srede [Maximum allowable concentrations of chemicals in the environment]. Saint-Petersburg, Mir I Semy'a, 2006, 360 p. (in Russian).

3. Camargo J.A., Alonso A. Ecological and toxicological effects of inorganic nitrogen pollution in aquatic ecosystems: $A$ global assessment. Environ. Int., 2006, vol. 32, pp. 831-849. doi:10.1016/j.envint.2006.05.002.

4. Swann P.F. The toxicology of nitrate, nitrite and n-nitroso compounds. J. Sci. Food Agric., 1975, vol. 26, no. 11, pp. $1761-1770$.

5. Fomin G.S. Voda. Kontrol khimicheskoi, bakterial 'noi i radiatsionnoi bezopasnosti po mezhdunarodnym standartam. Entsiklopedicheskii spravochnik [Water. Inspection of chemical, 
bacteriological and radiation safety according to international standarts]. Moscow, Protector, 2000, 849 p. (in Russian). 6. Mesquita R., Ferreira M., Segundo R., Teixeira C., Bordalo A., Rangel A. Development of a sequential injection system for the determination of nitrite and nitrate in waters with different salinity: Application to estuaries in NW Portugal. Anal. Methods., 2009, vol. 1, pp. 195-202. doi:10.1039/B9AY00101H. 7. Yue X-F., Zhang Z-Q., Yan H-T. Flow injection catalytic spectrophotometric simultaneous determination of nitrite and nitrate. Talanta, 2004, vol. 62, pp. 97-101. doi:10.1016/S00399140(03)00421-1.

8. Monser L., Sadok S., Greenway G.M., Shah I., Uglow R.F. A simple simultaneous flow injection method based on phosphomolybdenum chemistry for nitrate and nitrite determinations in water and fish samples. Talanta, 2002, vol. 57, pp. 511-518. doi: 10.1016/S0039-9140(02)00057-7.

9. Ayala A., Leal L. O., Ferrer L., Cerda V. Multiparametric automated system for sulfate, nitrite and nitrate monitoring in drinking water and wastewater based on sequential injection analysis. Microchem. J., 2012, vol. 100, pp. 55-60. doi:10.1016/j.microc.2011.09.004.

10. Ellis P.S., Shabani M.H., Gentle B.S., McKelvie I.D. Field measurement of nitrate and estuarine waters with a flow analysis system on-line zinc reduction. Talanta., 2011, vol. 84, pp. 98-103. doi: 10.1016/j.talanta.2010.12.028

11. Pons C., Santos J., Lima J., Forteza R., Cerda V. Multipumping flow system for the determination of nitrite and nitrate in water samples. Microchim Acta., 2008, vol. 161, pp. 73-79. doi. 10.1007/s00604-007-0822-z.

12. Legnerova Z., Solich P., Sklenarova H., Satinsky D., Karlicek R. Automated simultaneous monitoring of nitrate and nitrite in surface water by sequential injection analysis. Water Re- search, 2002, vol. 36, pp. 2777-2783. doi:10.1016/S00431354(01)00513-9.

13. Bulatov A.V., Ivasenko P.A., Moskvin L.N. [Simultaneous stepwise injection-photometric determination of nitrite and nitrate ions in aqueous media]. Zhurn. analit. khim., 2010, vol. 65. no. 8. pp. 833-837 (in Russia). [J. Anal. Chem., 2010, vol. 65, no. 8, pp. 816-819 (Engl. Transl.)]. doi.10.1134/S1061934810080095. 14. Kazemzadeh A., Ensafi A.A. Simultaneous determination of nitrite and nitrate in various samples using flow-injection spectrophotometric detection. Microchem. J., 2001, vol. 69, pp. 159-166. doi:10.1016/S0026-265X(01)00072-8.

15. Ensafi A.A., Rezael B., Nouroozi S. Simultaneous spectrophotometric determination of nitrite and nitrate by flow injection analysis. Anal. Sci., 2004, vol. 20, pp. 1749-1753.

16. Ensafi A.A., Kazemzadeh A. Simultaneous determination of nitrite and nitrate in various samples using flow injection with spectrophotometric detection. Anal. Chim. Acta., 1999, vol. 382, pp. 15-21. doi:10.1016/S0003-2670(98)00755-7. 17. Shpigun L.K. [Flow-injection analysis]. Zhurn. analit. khim. [J. Anal. Chem.], 1990, vol. 45, no. 6, pp. 1045-1091 (in Russian). 18. Mozzhukhin A.V., Moskvin A.L., Moskvin L.N. [Stepwise injection analysis as a new method of flow analysis]. $J$. Anal. Chem., 2007, vol. 62, no. 5, pp. 527-531. doi.10.1134/ S1061934807050152.

19. Klochkovskii S.P., Chistota V.D. [Catalytic method of the detection and quantity determination of nitrite-ions and nitrogen oxide.] Sbornik nauchnykh trudov MGMI [Collection of proc. of the Nosov MGMI], 1971, no. 87, pp. 70-72 (in Russian). 20. Bermudez B., Rios A., Luque de Castro M.D., Valcarcel $M$. Configuration with internally coupled valves to overcome shortcomings in the simultaneous determination of nitrite and nitrate by flow-injection analysis. Talanta, 1988, vol. 35, pp. 810-812. doi.10.1016/0039-9140(88)80190-5. 